\title{
Recherche sur contrat et collaborations internationales, et leur effet sur la recherche sinologique en France
}

\section{Vincent Goossaert}

L'importance grandissante de la recherche sur contrat est l'une des évolutions les plus importantes actuellement à l'œuvre dans la recherche française - évolution décidée par nos dirigeants et qui génère depuis plusieurs années un débat de fond dans l'ensemble du milieu scientifique. En marge de ce débat de fond, je me propose de lancer des pistes de réflexion sur les effets immédiats de la recherche sur contrat dans la sinologie française, dans un contexte d'expansion quantitative et qualitative de la sinologie dans le monde chinois, et en particulier en République populaire de Chine. Mon propos n'est pas la recherche sur contrat en général, mais les cas spécifiques où un (ou plusieurs) partenaire français est lié dans ce contrat à un (ou plusieurs) partenaire du monde chinois. Après avoir présenté deux types particulièrement importants de contrat - Agence nationale de la Recherche (ci après ANR) et la Chiang Ching-kuo Foundation for International Scholarly Exchange (ci après $\mathrm{CCKF}$ ) -, je voudrais évoquer certains de leurs effets : 1) nos rapports avec les collègues chinois (ce que nous pouvons leur apporter et réciproquement ; ce qu'ils nous demandent et réciproquement) ; 2) les rapports entre logiques de projet et logiques de laboratoires ; 3) les résultats attendus de ces projets, notamment en termes de publications ; 4) les nouvelles possibilités et contraintes pour le financement des doctorants et post-doctorants.

Les réflexions qui suivent découlent en large partie de mon expérience (en cours à l'heure où j'écris ces lignes) de direction d'un projet de recherches intitulé « Temples, Urban Society, and Taoists: The Transfor- 
mation of Modern Urban Chinese Religious Organizations » (2007-2010). Ce projet vise à conjuguer les approches de l'histoire moderne (travail dans les archives, manuscrits, journaux, etc.) et de l'anthropologie pour comprendre les évolutions et adaptations du taoïsme en milieu urbain depuis la fin du XIX ${ }^{\mathrm{e}}$ siècle, en comparant plusieurs grandes villes, notamment Hangzhou, Wuhan et Guangzhou. Ce projet est financé par l'ANR et la CCKF, et comprend des collègues français (trois chercheurs ou ingénieurs CNRS et une doctorante), américain (un chercheur), taiwanais (un chercheur), hong-kongais (deux chercheurs et un assistant) et chinois (trois chercheurs). Outre des réunions de travail annuelles, le projet organisera un colloque final à Tainan fin 2010 et publiera trois volumes de matériaux en chinois et un volume de synthèse en anglais, ainsi qu'une série d'articles individuels ou collectifs ${ }^{1}$.

\section{Le financement des projets}

Dans un contexte bien connu où les crédits récurrents des équipes de recherche sont souvent en stagnation, la recherche en sinologie, et tout particulièrement les missions sur le terrain, nécessitent de trouver des financements sur contrat. De fait, les missions constituent le plus souvent l'essentiel des budgets de ces contrats, que ce soit pour l'archéologie, la linguistique, l'histoire moderne, l'anthropologie ou d'autres sciences sociales. Ceci est vrai pour l'ensemble des chercheurs (terme dans lequel j'englobe ici les universitaires, chercheurs CNRS, EFEO et autres, sans distinction), et sans doute particulièrement pour ceux qui travaillent au sein d'équipes de recherche qui ne sont pas entièrement consacrées à la Chine ou à l'Extrême-Orient, et dont les besoins de mission paraissent particulièrement importants au regard de collègues travaillant sur des aires plus proches de la France.

Les sources de financement, effectives ou potentielles, pour des projets de recherche sinologiques sont nombreuses : collectivités locales (qui depuis une dizaine d'années augmentent considérablement leur budget d'aides à la recherche), programmes divers du CNRS comme les PEPS (Projets exploratoires/premier soutien), du ministère en charge de la Recherche et des autres pouvoirs publics ; programmes européens, fondations privées, etc. Ces sources diverses proposent une gamme allant de petits

1 http://www.gsrl.cnrs.fr/taoist-and-temple, consulté le 22 mars 2010. 
projets individuels (tels que les PEPS) jusqu'aux montages les plus lourds (Union européenne), même si, à ma connaissance, un seul projet sinologique français a obtenu un financement communautaire récemment ${ }^{2}$, dans le cadre du Conseil européen de la recherche (ci après ERC de son acronyme anglais) : « The hybrid syntactic typology of Sinitic languages » (2009-2012) dirigé par Hilary Chappell (EHESS, CRLAO).

Les deux sources de financement les plus sollicitées sont l'ANR et la CCKF. Elles présentent des traits communs, notamment celui de financer essentiellement des projets sur trois ans (et il arrive qu'un même projet soit soumis aux deux instances), même si les règles administratives (possibilité de payer un salaire par exemple) ne sont pas les mêmes. Tandis que la CCKF est une structure assez souple et légère (tant pour la soumission que pour le suivi), le montage d'un projet ANR est une opération compliquée ; certaines équipes ont accès à des ingénieurs-conseils (CNRS ou universitaires) spécialisés. Leur aide est extrêmement précieuse pour répondre aux appels d'offre, dont les volets financiers sont remarquablement ésotériques, et dont même les volets scientifiques demandent une compréhension des mécanismes d'évaluation. Dans tous les cas, la préparation d'une soumission nécessite un gros travail en amont, idéalement sur une année, tant pour déterminer un budget réaliste et fiable (auquel les partenaires seront tenus par la suite ${ }^{3}$ ) que pour élaborer un calendrier de travail précis.

\section{L'ANR}

Les financements de l'ANR, depuis 2005, se font sur appels à projets, soit thématiques, soit «blancs » (ouverts à tous). À ce jour, aucun appel à projet thématique spécifique sur la Chine n'a été émis ; on trouve cependant chaque année plusieurs chercheurs sinologues parmi les projets retenus, soit au sein de projets plus larges, comparatifs ou disciplinaires, soit au sein de

2 Rappelons que le «Projet Tao-tsang 》 (1978-1984) dirigé par Kristofer Schipper qui rassemblait des sinologues français, allemands, anglais et italiens fut l'un des premiers à bénéficier de financements de l'European Science Foundation.

3 Mon expérience d'une soumission de projet à Taiwan suggère que la contrainte du budget a priori y est plus importante encore : il faut une autorisation spécifique pour utiliser les fonds à une dépense non initialement prévue dans le contrat. 
projets explicitement sinologiques. Parmi ceux-ci, les archives en ligne de l'ANR ${ }^{4}$ permettent d'identifier les projets suivants, qui ne constituent certainement pas une liste exhaustive : «Nouvelles formes d'association, nouvelles figures des liens sociaux, nouvelles définitions de l'intérêt général : les modes de réorganisation de la société chinoise contemporaine », Isabelle Thireau (2005) ; « International Trading Hubs in East and Southeast Asia », François Gipouloux (2005) ; « Dynamique des salaires et globalisation en Chine », Sandra Poncet (2006) ; « Reconstitution des systèmes de la pluralité et du nombre dans le sino-tibétain », Xu Dan (2006), « Temples, Urban Society, and Taoists: The Transformation of Modern Urban Chinese Religious Organizations », Vincent Goossaert (2007) ; " Contacts de langues et changements linguistiques : cas du chinois et des langues altaïques », Redouane Djamouri (2007) ; " Diachronic change in Southern Min, a Sinitic language - Changement diachronique en min méridional, langue sinitique », Hilary Chappell \& Alain Peyraube (2008) ; « Système d'informations numériques de l'emploi des textes dans l'ornementation des monuments bouddhiques : expérimentation sur la tombe de l'empereur Qianlong », Françoise Wang-Toutain (2008) ; « Loin d'Hollywood. Histoire culturelle du cinéma en France, URSS, Chine, 1927-1933 », Dimitri Vezyroglou (2008); « What defines Qiang-ness? Towards a phylogenetic assessment of the Southern Qiangic languages of Muli », Guillaume Jacques \& Katia Chirkova (2008), «Chine rurale : le village de Lianmin/Base de données électronique (19491999) », Isabelle Thireau (2009) ; " Itinéraires individuels et circulation des savoirs scientifiques et techniques en Chine moderne ( $\mathrm{XVI}^{\mathrm{e}}-\mathrm{XX}^{\mathrm{e}}$ siècle) », Catherine Jami (2009).

Si quelques-uns de ces projets ont été financés sur des appels thématiques, la grande majorité l'a été sur des appels «blancs » ouverts à tous. Même si le taux de rejet des appels d'offre de l'ANR est relativement élevé (l'ordre de grandeur des projets retenus semble être de $20 \%$ ), et que la recherche sur la Chine n'y occupe pas une place visible, on note tout de même que la vitalité de la sinologie française permet d'y obtenir en moyenne deux contrats par an. Une remarque positive est que, même si l'inquiétude de la communauté scientifique quant à la polarisation de la recherche sur les sujets « à la mode », médiatisés, ou portés par les politiques reste une question de fond dans les débats autour de l'ANR, on peut

\footnotetext{
${ }^{4}$ http://www.agence-nationale-recherche.fr, consulté le 22 mars 2010.
} 
constater que les projets retenus, notamment dans les appels « blancs » ne sont pas des sujets « à la mode » mais simplement de vrais projets intellectuels qui ont été évalués en tant que tels, ce qui est rassurant, même si cela ne constitue en rien une garantie pour l'avenir.

\section{La Fondation Chiang Ching-kuo}

Les projets de recherche financés (depuis 1989) par la fondation Chiang Ching-kuo (CCKF) répondent davantage à un modèle « léger » (un seul chercheur occidental titulaire suffit, souvent accompagné d'un ou plusieurs doctorants ou post-doctorants et de partenaires chinois) du projet de recherche, par contraste avec le modèle ANR qui nécessite une « masse critique » de chercheurs titulaires. La gestion de la CCKF se fait par région (Taiwan, Asie-Pacifique, Europe, et Amériques), et les types de financements disponibles ainsi que les processus d'évaluation diffèrent d'une région à l'autre. Pour la région Europe, huit types de financements sont offerts : développement institutionnel ; séries de conférences ; projets de recherche ; aides aux colloques ; aides aux publications ; soutiens aux chercheurs confirmés ; bourses de thèse et postdoctorales ; bourses de thèse pour étudiants taiwanais en Europe ${ }^{5}$. Si je m'intéresse ici essentiellement aux financements des projets de recherche, il faut noter qu'un projet bénéficiant d'un financement CCKF au titre de la recherche peut aussi demander séparément à la fondation une aide pour organiser un colloque ou financer une publication.

Parmi les projets de recherche qu'elle a financés depuis 2004, les archives en ligne de la fondation ${ }^{6}$ permettent d'identifier les projets suivants dirigés par des chercheurs basés en France : "The Common People and the Artist in the 1930s: An Essay in Chinese Cultural and Social MetaHistory through the Visual Sources », Christian Henriot (2004) ; "The Temples of Peking: Epigraphy and Oral Sources - The Social History of an Imperial Capital », Marianne Bujard (2004) ; « Social and Cultural History of Printing and Publishing in Huizhou », Michela Bussotti (2005) ; «Professional Cultures and the Transmission of Specialized Knowledge:

5 http://www.cckf.org.tw, consulté le 22 mars 2010.

${ }^{6}$ http://www.agence-nationale-recherche.fr, consulté le 22 mars 2010. 


\section{Vincent Goossaert}

Artisans and Merchants in Local Society », Christian Lamouroux (2006); « Temples, Urban Society, and Taoists: The Transformation of Modern Urban Chinese Religious Organizations », Vincent Goossaert (2007) ; « Religion, Society, and the Economy in Huizhou (Anhui) », John Lagerwey (2008) ; " Languages in Contact in Northwestern China: Convergence, Mixed Languages or Linguistic Area?», Alain Peyraube (2008) ; «Lianmin Village/Zhang Letian Electronic Database (1949-1999)», Isabelle Thireau (2009) ; " The "Confucian Revival" in Mainland China: Forms and Meanings of "Confucian Piety" Today », Sébastien Billioud (2009).

On remarque ainsi que chaque année, un ou deux projets portés par une institution française sont financés, et que la sinologie française tient donc une place tout à fait significative au sein la zone Europe et plus largement dans la sinologie mondiale. Ceci est tout à fait encourageant, non seulement en termes d'accès aux financements, mais aussi pour le « label de qualité » que représente un financement CCKF dans la communauté sinologique, en particulier dans certains domaines comme l'anthropologie ou l'histoire. On constate encore que si l'importance qu'occupent depuis longtemps les recherches sur la société locale traditionnelle (orientées vers la collecte de l'histoire orale et de manuscrits) dans les financements de la CCKF ne se dément pas vraiment, les financements vont aussi à des projets plus purement historiques et comparatifs, mais aussi linguistiques.

\section{La collaboration internationale}

En bref, les financements réguliers obtenus par les projets de recherche sinologiques français, même s'ils restent en deçà des besoins, et si chaque année d'excellents projets sont recalés, donnent une image positive de la recherche en France, reconnue internationalement comme innovante. Reste à se demander ce que ces projets sur contrats font à la recherche sinologique en France, en particulier dans le cadre de collaborations formelles avec les collègues du monde chinois (en RPC, à Taiwan, et à Hong Kong). Si les différents types de financements correspondent à des gestions de projets différentes, je voudrais esquisser ici quelques questions générales en les considérant dans leur ensemble.

Même si elles prennent des formes diverses (les institutions chinoises ne sont que des « prestataires de services » dans un projet ANR, tan- 
dis qu'ils sont des partenaires à part entière dans un projet CCKF), les collaborations avec les collègues chinois sont simplement indispensables à un projet sinologique. Elles soulèvent aussi des questions, tant politiques que financières. Les questions politiques, qui peuvent aisément rendre le travail d'un directeur de projet extrêmement compliqué, touchent d'abord à la propriété des données et leur droit d'accès ou de reproduction (sites et matériel archéologiques, données d'enquête, manuscrits et livres rares). Ces questions qui peuvent souvent se résoudre assez simplement pour un individu rassemblant des données en vue d'une publication personnelle en langue occidentale, deviennent plus complexes à négocier dans le cadre d'un projet formalisé et financé. À cela s'ajoute parfois des problèmes liés à l'affichage des partenaires taiwanais en RPC.

Les aspects financiers de la collaboration ne sont pas plus simples. Maintenant que les plus grandes universités chinoises sont devenues des institutions très riches (mais aussi très endettées), le contexte financier des échanges et collaborations est devenu totalement différent de ce qu'on a pu connaître dans les années 1980 et 1990. Là où jadis les invitations à participer à un colloque ou de venir comme professeur invité pendant un mois constituaient le premier élément de l'offre du côté français, les choses sont devenues plus complexes ; certaines universités chinoises proposent maintenant aux collègues européens des postes de professeurs invités avec des salaires nettement supérieurs à ceux en vigueur en France... Mais, outre l'intérêt scientifique à la source de toutes les collaborations réelles, les collègues chinois restent malgré tout demandeurs de projets où les partenaires français apportent aussi un financement.

L'une des formes que cela prend est que les collègues chinois, comme ceux de certaines institutions scientifiques européennes ou nord-américaines, sont en partie évalués sur leur capacité à apporter des contrats et des financements. L'apport entre parfois dans le cadre de « matching funds »: l'université partenaire s'engage à mettre dans un projet une somme équivalente à celle apportée par le partenaire occidental. Ces «matching funds » sont mis en place dans un contexte où certaines universités ont des problèmes de trésorerie et cherchent à attirer de l'argent extérieur. En revanche, les prélèvements pour frais de gestion (connus lorsqu'ils sont redistribués sous le terme de BQR : Bonus 


\section{Vincent Goossaert}

qualité recherche) dans les universités françaises (souvent à un taux de $9 \%$, et que le CNRS applique aussi au taux de $9 \%$ ) restent plus modestes dans les universités chinoises, et sont parfois nuls. Par ailleurs, il est très possible que les partenaires chinois (comme les partenaires français) utilisent le travail de préparation et le crédit (au sens symbolique) d'un financement étranger pour obtenir un financement chinois pour le même projet, ou pour un projet lié. Si cette multiplication des financements est une bonne chose, elle oblige aussi, au cours d'un projet où le temps presse dès le premier mois, à jongler entre les échéances et les exigences propres à chacun des bailleurs de fonds.

Certes, ces différentes questions ne relèvent pas des aspects scientifiques de la recherche, mais il est préférable de les comprendre avant de mettre en place une collaboration formelle. Si l'estime et la confiance réciproques restent de très loin les facteurs les plus déterminants dans la réussite d'une collaboration, la compréhension de la culture institutionnelle de l'autre est aussi un élément important. Dans de nombreux projets, les questions financières sont dans une large mesure évacuées en divisant d'emblée le financement entre les partenaires qui gèrent chacun leur part de façon autonome ; une telle approche peut cependant entrer en contradiction avec le besoin de comparer les différents terrains et d'avoir une vraie démarche collective.

\section{Les effets dans les équipes de recherche}

Le fait de gérer un projet avec un budget propre induit donc des effets spécifiques dans les rapports avec les collègues chinois. Il induit aussi, pour des raisons en partie semblables, des effets au sein de l'équipe de recherche en France même, une question qui est souvent difficile à comprendre pour nos collègues (aussi bien chinois qu'européens et nord-américains) qui ne connaissent pas le modèle très collectif de la recherche en SHS (sciences humaines et sociales) en France. Le chercheur français est en effet tenu d'être solidaire de son équipe, or, pour son projet, il doit mettre en place une communication (site Internet, publications) propre au projet; d'où parfois des stratégies de publication différenciées (articles de synthèse en français ; articles «fouillés » en chinois ou anglais). Il peut être invité à organiser des manifesta- 
tions dans son équipe; or organiser un colloque international avec un bon nombre de collègues chinois est considérablement plus simple et meilleur marché en RPC ou à Hong Kong. De façon générale, le responsable de projet est moins disponible à son équipe alors qu'il est plus exposé.

Les aspects financiers d'un projet sur contrat soulèvent aussi des questions spécifiques - voire peut-être (même si je n'en connais pas de cas) des tensions dans une équipe où un projet avec un seul chercheur peut disposer sur trois ans d'autant de fonds que l'ensemble de l'équipe. La gestion financière d'un contrat, avec ses contraintes spécifiques (rajoutées à celles de la comptabilité publique française), constitue une surcharge de travail pour les gestionnaires des équipes de recherche dont on connaît la situation de sous-effectif. L'ANR prévoit une dotation au titre de frais de gestion pour l'équipe gestionnaire, mais pas la CCKF.

Cependant, l'évaluation des équipes de recherche (non seulement par l'AERES mais aussi en interne par le CNRS et les universités) tendant à valoriser toujours plus l'obtention de contrats, le bénéfice pour une équipe de voir l'un de ses membres gérer un contrat l'emporte de très loin sur tout inconvénient; cela crée simplement un contexte de travail très spécifique pour un directeur de projet dont ce dernier doit tenir compte dans son rapport avec les collègues français.

\section{Les effets sur la publication de la recherche}

L'un des effets des recherches sur contrat est d'inciter (pour ne pas dire obliger) à la publication rapide de résultats ; l'évaluation a posteriori y est en effet plus exigeante que celle des financements CNRS ou universitaires. Elle pousse aussi à la publication non seulement en français, mais aussi en anglais et chinois. On peut estimer (c'est en tout cas mon avis) que c'est un effet positif; les recherches menées en France gagnent ainsi l'audience qu'elles méritent à l'étranger. Les financements de la CCKF, et la collaboration avec les chercheurs chinois, poussent en particulier à publier en chinois, à la fois les matériaux bruts (comptes rendus de terrain ; textes rares tels que manuscrits ou archives ; images) et des volumes d'essais. Cela correspond à la fois à une demande des institutions de financement et des partenaires chinois, même si les publications en langues occidentales 


\section{Vincent Goossaert}

comptent dans l'évaluation des chercheurs à Taiwan et Hong Kong, et de façon plus inégale (et depuis quelques années seulement) en RPC. Un projet collaboratif doit donc avoir une stratégie de publication (livres/articles, chinois/anglais/français ; données/théorie) qui satisfasse les désirs et les besoins (en termes d'évaluation et de carrière) des partenaires chinois aussi bien que français et autres. La publication rapide des résultats entre de plus dans le modèle de la recherche sur contrat telle qu'elle se pratique en RPC, à Taiwan et à Hong Kong, qui exige des publications très (voire, selon nos habitudes, extraordinairement) rapides. Il est fréquent de voir les collègues de RPC obtenir un financement (parfois tout à fait conséquent) pour un livre devant être effectivement publié au bout de 24 mois.

Gérer un projet en collaboration avec des collègues chinois implique de comprendre cette façon de faire les choses, car c'est là le modèle dans lequel ils ont l'habitude de travailler. Aussi, idéalement, un directeur de projet, s'il n'est pas lui-même issu de l'université chinoise, doit pouvoir travailler au quotidien en France avec quelqu'un qui comprend le système universitaire chinois de l'intérieur, ce qui implique concrètement d'y avoir été formé. Or, de façon générale, les chercheurs formés en RPC et titulaires en France restent trop peu nombreux et ce manque joue de façon défavorable dans la gestion des collaborations universitaires formelles, non pas seulement au niveau intellectuel, mais aussi au niveau de la culture des organisations.

La publication en langue chinoise des chercheurs français est relativement fréquente (notamment sous forme de traductions de travaux déjà publiés) ; les publications collectives de résultats inédits est moins courante, sauf sans doute en linguistique et en archéologie ${ }^{7}$. Cette tendance à la publication accrue en chinois est évidemment une excellente chose ; il faut noter qu'elle implique (outre la question des délais) trois contraintes. La première est de disposer au sein du projet d'un éditeur scientifique rompu aux publications en langue chinoise, à trouver soit chez les partenaires chinois, soit à recruter sur contrat. De plus, il est fréquent que les textes des partenaires français soient d'abord écrits en français ou anglais puis traduits; or si les

7 Voir par exemple Qin Jianming 秦建明 \& Marianne Bujard 吕敏, Yaoshan shengmu miao yu shenshe 堯山聖母廟與神社, Beijing : Zhonghua shuju, 2003 et les autres publications du projet « Hydraulique » piloté par l'EFEO ; et la collection Kejia chuantong shehui congshu 客家傳統社會叢書 dirigée par John Lagerwey. 
traducteurs abondent, les bons traducteurs sont beaucoup plus rares (et plus chers).

La seconde contrainte est celle, déjà évoquée, des délais, qui nécessitent une gestion fine : il est de plus en plus difficile en comptabilité publique française d'utiliser ses crédits au-delà de la date d'échéance du contrat, mais il est tout aussi difficile d'avoir terminé les publications et payé la maison d'édition à cette date... La logique de l'ANR (la CCKF voyant les choses assez différemment) est que la gestion d'un projet permet précisément d'acquérir des compétences managériales, mais ce n'est généralement pour cette raison-là qu'un chercheur s'engage à diriger un projet. Quoi qu'il en soit, un responsable de projet doit prendre en compte cette dimension et commencer à planifier précisément le calendrier du travail d'écriture et d'édition dès la première réunion de travail, ce qui n'est pas trop dans l'habitude des chercheurs français.

La troisième contrainte est le coût des publications. Si le fait que la publication d'un ouvrage scientifique collectif en français est devenue quasiment toujours payante (on parle couramment de 3 à 4000 euros) est à peu près entré dans les mœurs (et donc dans les budgets des équipes de recherche, encore que les politiques des équipes divergent sur ce point), la publication en langue chinoise est souvent bien plus coûteuse encore (du moins en termes relatifs), et plus systématiquement facturée aussi (pour des livres d'auteur, et même dans certaines revues). Si des maisons d'édition en RPC publient parfois sans subvention, en fonction du marché, de leur propre stratégie ou du jeu des relations personnelles, la demande de subvention y est quand même la norme, d'autant plus que le nombre de livres publiés est limité par des quotas. L'ordre de grandeur des prix en vigueur en 2010 pour un livre scientifique est de $1000 \mathrm{RMB}$ pour 10000 caractères, soit quelque 3000 euros pour un livre de 400 pages. La négociation avec les maisons d'édition (y compris les plus renommées) est essentiellement économique, le contenu scientifique étant (sauf à aborder de front des sujets sensibles) aisément accepté. Quand les budgets sont serrés, la tentation peut être grande de publier à Taiwan ou Hong Kong, où l'on trouve des maisons d'édition pouvant accepter pour un projet qui les intéresse de publier des ouvrages sans subvention, mais à l'heure actuelle, ces ouvrages (même purement scientifiques et sans portée d'actualité) restent très difficiles à obtenir en RPC et les collègues chinois ne préfèrent pas cette solution. 


\section{Emploi scientifique}

L'un des avantages les plus marqués des projets de recherche financés sur contrat ANR est la possibilité de financer une thèse (cela est possible sur certains contrats ANR mais pas sur d'autres) ou de recruter un postdoc. Cela est souvent impossible sur un contrat CCKF, mais la fondation offre par ailleurs des programmes de bourses. Certaines institutions de financement de la recherche à l'étranger (Allemagne, Belgique, etc.) sont d'ailleurs formatées de manière à financer des études postdoctorales pour des projets déposés par leur tuteur. On voit là que l'ANR qui ne sépare pas l'emploi scientifique des projets collectifs reste sur un modèle largement français, en dépit des critiques qui lui ont été adressées de se conformer à un « modèle anglo-saxon » qui n'existe pas en fait.

Au-delà du débat sur la précarisation de l'emploi scientifique, la possibilité de recruter un post-doctorant, ne serait-ce que pour un an, est une joie pour un chercheur qui fréquente quotidiennement des jeunes chercheurs sans poste, surtout que ces postes sur contrat ne sont pas des concours, mais des recrutements discrétionnaires. Le problème du choix se pose cependant. La plupart des postes de post-doctorat existants (très rares en France, ceux du CNRS ayant fort malheureusement été supprimés en 2009) consistent à salarier un jeune chercheur pour travailler (outre, éventuellement un peu d'enseignement) sur son propre projet. Or, un projet collectif nécessite que le post-doctorant travaille d'abord sur la publication collective, et seulement secondairement sur la publication de sa thèse. Par ailleurs, les projets demandent souvent des compétences techniques (outils informatiques) ou linguistiques (éditer des matériaux ou un volume en chinois) que n'ont pas les étudiants sortant des universités françaises, ce qui peut mener les projets financés en France à recruter un post-doctorant chinois, qui peut travailler en France ou ailleurs.

Enfin, même sans possibilité de fournir un contrat de travail à un doctorant ou un post-doctorant, il faut aussi réaliser qu'un contrat permet de leur financer des missions de terrain assez longues en RPC ou à Taiwan, ce qui s'avère très souvent impossible sur les crédits propres des équipes de recherche, alors même que les doctorants ou post-doctorants n'ont pas les moyens de les financer par eux-mêmes. 
En conclusion, un projet de recherche sur contrat en collaboration avec des partenaires chinois est un type d'expérience scientifique spécifique, qui a ses effets propres sur la recherche en France. Elle permet, bien plus que les collaborations amicales et individuelles, voire même que les collaborations ponctuelles comme l'organisation d'un colloque (et de façon sans doute différente des accords universitaires d'échanges d'enseignants et étudiants), de comprendre vraiment, y compris par l'erreur et les impasses, comment fonctionne le monde universitaire chinois - et accessoirement, d'élargir sa compréhension du monde universitaire français par la même occasion. C'est une expérience riche, frustrante parfois, fascinante aussi, et hautement formatrice. On ne peut que se réjouir de voir de nombreux sinologues français s'y engager avec d'excellents résultats. 